\title{
Paradoxical Hypocapnia While Wearing a Facemask: A Novel Cause of Orthostatic Intolerance - A Case Report
}

\section{C. (Linda) M.C. van Campen $^{1} \mid$ Peter C. Rowe $^{2}$ | Frans C. Visser ${ }^{1 *}$}

\section{*Correspondence: Frans C Visser}

Address: ${ }^{1}$ Stichting CardioZorg, Planetenweg 5, 2132 HN Hoofddorp, Netherlands; ${ }^{2}$ Department of Pediatrics, Johns Hopkins University School of Medicine, Baltimore, MD, USA

e-mail $\bowtie$ : fransvisser@stichtingcardiozorg.nl

Received: 24 June 2021; Accepted: 05 July 2021

Copyright: (C) 2021 Van Campen CMC. This is an open-access article distributed under the terms of the Creative Commons Attribution License, which permits unrestricted use, distribution, and reproduction in any medium, provided that the original work is properly cited.

\section{ABSTRACT}

Previous studies with facemasks have shown significant respiratory effects, including increased breathing resistance, $\mathrm{CO} 2$ rebreathing due to $\mathrm{CO} 2$ accumulation in the facemask cavity leading to hypercapnia, and decreased inhaled 02 concentration. These changes may lead to a number of symptoms like dyspnea, headache, sweating, and dizziness. In the present case report a patient is described with similar symptoms while wearing a facemask, but with the opposite effect: a hyperventilatory hypocapnic response, leading to orthostatic intolerance symptoms.

A 34 year old female patient was examined with symptoms while wearing a facemask in public. She was studied in the supine position. First, end-tidal $\mathrm{CO} 2$ and cerebral flow measurements, using extracranial Doppler imaging, were obtained without facemask. Thereafter, after 10 minutes of wearing a facemask, measurements were repeated.

While wearing the facemask, the end-tidal $\mathrm{CO} 2$ decreased from $35 \mathrm{mmHg}$ without the facemask to $28 \mathrm{mmHg}$ with the facemask, respiration rate increased from 10 to 15 breath/min and cerebral blood flow decreased from 565 $\mathrm{ml} / \mathrm{min}$ to $419 \mathrm{ml} / \mathrm{min}$, a $26 \%$ reduction while wearing the facemask. These changes were accompanied by her typical orthostatic intolerance symptoms while wearing the facemask in public

As shown in this patient, wearing a facemask cannot only cause hypercapnia, as has been previously described, but may also cause hypocapnia, leading to a cerebral blood flow reduction and orthostatic intolerance symptoms. Keywords: Facemask, Extracranial Doppler Imaging, Cerebral Blood Flow, End-Tidal CO2, Hypocapnia

\section{Introduction}

In the SARS-CoV2 era, some people experience dyspnea, dizziness, palpitations and a general feeling of discomfort while wearing a facemask in public areas, symptoms that are not present without wearing the facemask. Previous studies have shown that facemasks can exert significant respiratory effects, including increased breathing resistance, $\mathrm{CO} 2$ rebreathing due to $\mathrm{CO} 2$ accumulation in the 
facemask cavity, and decreased inhaled 02 concentration (Johnson, 2016). CO2 rebreathing can result in hypercapnia and lead to dyspnea, headache, sweating, and dizziness, even in individuals without medical diseases (Johnson, 2016). Similar symptoms can also accompany orthostatic intolerance (van Campen et al., 2020), often in the presence of hypocapnia. We evaluated a patient whose symptoms occurred while wearing a facemask. To assess whether symptoms were associated with hyper- or hypocapnia, we measured cerebral blood flow (CBF) measurements using extracranial Doppler, in combination with endtidal CO2 pressure (PetCO2) assessment with and without wearing a facemask.

\section{Case Report}

A 34 year old female patient with previously diagnosed myalgic encephalomyelitis/chronic fatigue syndrome (ME/CFS) and proven orthostatic intolerance developed symptoms acutely after putting on a facemask in shops. She experienced immediate onset of an increased respiratory rate, followed by a tingling feeling in the head, increased tension in the shoulder muscles radiating to the neck and head, and an increased pressure sensation within the head. She often reported associated chest pressure and nearsyncope. Within 3 minutes she usually had to remove the facemask. Doing so was associated with a gradual relief of symptoms. Her facemask is commercially available, custom-made, consisting of three layers of $100 \%$ cotton, without a filter.

To evaluate her symptoms, we measured cerebral blood flow (CBF) by extracranial Doppler of the internal carotid and vertebral arteries, using a Vivid-I system (GE Healthcare, Hoevelaken, the Netherlands). PetCO2, heart rate, respiration rate and 02 saturation were measured using a Lifesense device (Nonin Medical, Minneapolis USA). First, measurements were performed in the supine position, with the facemask off, after 10 minutes of resting. Then, the mask was put on and measurements were repeated in the supine position after 10 minutes. The methodology to measure CBF have been described previously (van Campen et al., 2020). In short, high resolution B mode images, color Doppler images and the Doppler velocity spectrum (pulsed wave mode) were recorded in one frame of the two internal carotid (ICA) and the two vertebral arteries (VA). Blood flow in the individual vessels was calculated from the mean blood flow velocities $x$ the vessel surface area. CBF was the sum of the 4 vessels.

Without the facemask, her mean PetCO2 was $35 \mathrm{mmHg}$, heart rate $72 \mathrm{bpm}$, respiratory rate 10 breaths per min, and 02 saturation 97\%. While wearing the face mask the PetCO2 gradually decreased to $28 \mathrm{mmHg}$, heart rate gradually increased to $83 \mathrm{bpm}$, respiration rate increased to $15 \mathrm{breaths} / \mathrm{min}$ and 02 saturation increased to $98 \%$. After $10 \mathrm{~min}$ of wearing the facemask she developed the typical complaints she experiences while shopping. 
Fig. 1 shows the flow velocity profiles with extracranial Doppler of the 2 carotid and 2 vertebral arteries without facemask (left panels) and with facemask (right panels). Flow in the left ICA decreased from $174 \mathrm{ml} / \mathrm{min}$ without facemask to $121 \mathrm{ml} / \mathrm{min}$ while wearing the mask, in the right ICA from 219 to $174 \mathrm{ml} / \mathrm{min}$, in the left VA from 56 to $40 \mathrm{ml} / \mathrm{min}$, in the right VA from 116 to $84 \mathrm{ml} / \mathrm{min}$. Total CBF without the facemask was $565 \mathrm{ml} / \mathrm{min}$, and with facemask was $419 \mathrm{ml} / \mathrm{min}$, a 26\% CBF reduction while wearing the facemask. Table 1 shows the results of the mean flow velocities in the 4 arteries supine without facemask (left column) and supine with facemask (right column).
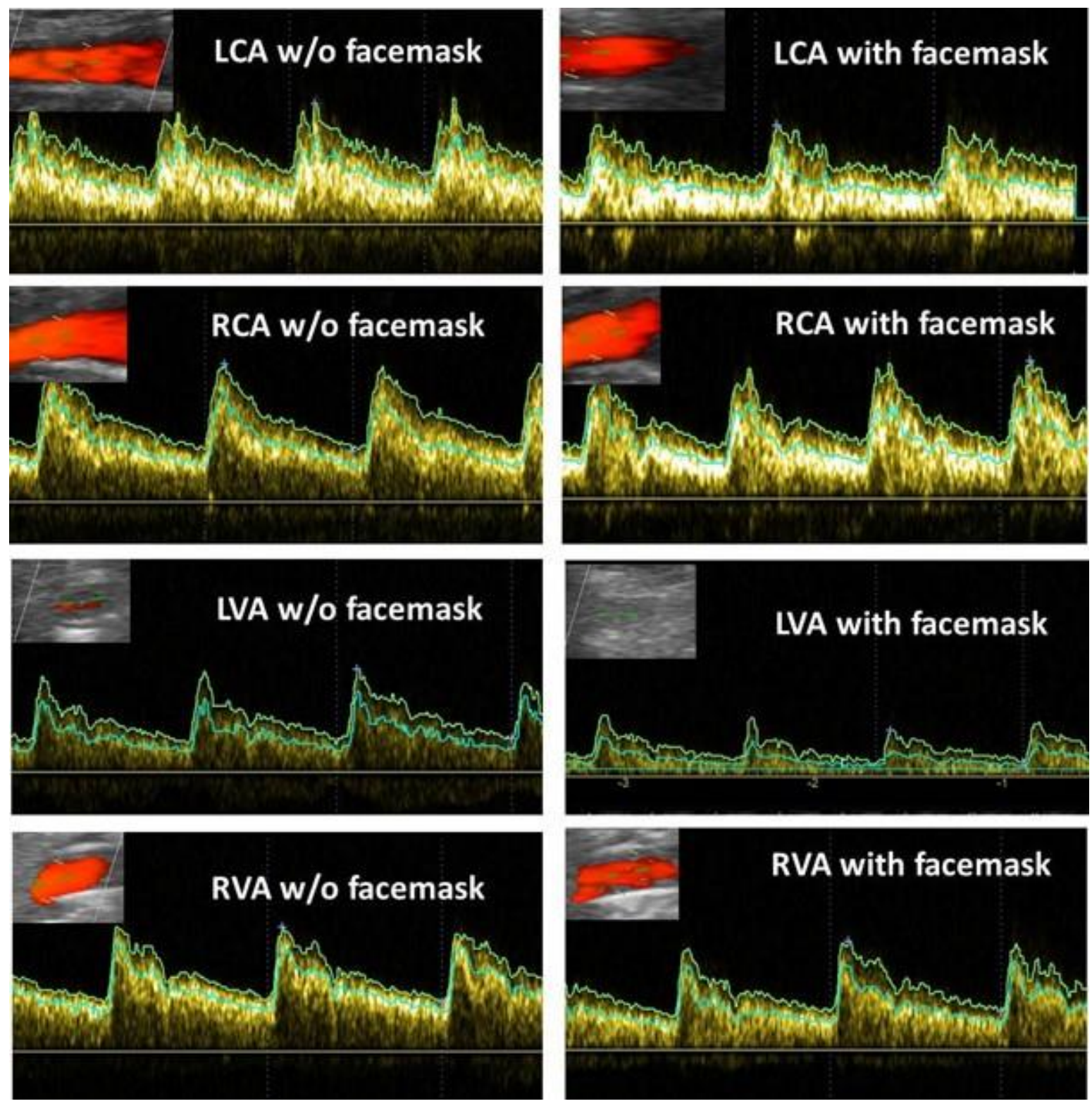

Figure 1: Cerebral arteries and their flow velocity profiles

Doppler flow velocity profiles are shown in the figure for each vessel. Vessel flows are shown in the inserts top left. The upper light green line in the velocity profiles indicate the peak flow velocities, the green lines below indicate the mean flow velocities. LCA: left carotid artery; RCA: right carotid artery; LVA: left vertebral artery; RVA: right vertebral artery. 
Table 1: Mean flow velocity measurements supine without and with facemask

\begin{tabular}{|l|l|l|}
\hline Supine mean flow velocities (cm/sec) & Without Facemask & With Facemask \\
\hline Left Carotid Artery (LCA) & 26.72 & 21.96 \\
\hline Right Carotid Artery (RCA) & 34.24 & 19.99 \\
\hline Left Vertebral Artery (LVA) & 19.02 & 12.61 \\
\hline Right Vertebral Artery (RVA) & 26.6 & 19.21 \\
\hline
\end{tabular}

\section{Discussion}

Prior research suggests that symptoms associated with wearing a facemask are related to a reduction in 02 uptake and/or increased PaCO2 (Perna et al., 2020). The primary response of our patient was a hypocapnic hyperventilatory response while wearing the facemask: 02 saturation and respiration rate increased, and PetCO2 decreased (Stewart et al., 2018).

Many studies have shown that CO2 changes profoundly change cerebral flow and vasoreactivity: see for an extensive review Hoiland, et at. (2011). A PaCO2 reduction leads to vasoconstriction of the intracranial arteries and thus to reduction of CBF and development of orthostatic intolerance symptoms, as shown in this patient (Hoiland et al., 2011).

Clinical Implications: Wearing a facemask cannot only cause hypercapnia, but, as shown in this patient, may also cause hypocapnia. The mechanism of the hypocapnia as well as the prevalence of this response and whether it is associated with orthostatic intolerance all remain to be studied.

\section{Conflicts of Interest: None for all authors}

Funding: No funding

Ethics Statement: The study was conducted according to the guidelines of the Declaration of Helsinki, and approved by the Institutional Review Board (or Ethics Committee) of the Slotervaart Hospital (protocol code P1411 and date of approval 14 march 2014).

\section{References}

Hoiland RL, Fisher JA, Ainslie PN. Regulation of the cerebral circulation by arterial carbon dioxide. Compr Physiol 2011; 9: 1101-1154.

Johnson AT. Respirator masks protect health but impact performance: a review. J Biol Eng 2016; 10: $1-2$.

Perna G, Cuniberti F, Daccò S, Nobile M, Caldirola D. Impact of respiratory protective devices on respiration: Implications for panic vulnerability during the COVID-19 pandemic. J Affect Disord 2020; 277: 
772-778.

Stewart JM, Pianosi P, Shaban MA, Terilli C, Svistunova M, Visintainer P, Medow MS. Postural hyperventilation as a cause of postural tachycardia syndrome: increased systemic vascular resistance and decreased cardiac output when upright in all postural tachycardia syndrome variants. J Am Heart Assoc 2018; 7: e008854.

van Campen CL, Verheugt FW, Rowe PC, Visser FC. Cerebral blood flow is reduced in ME/CFS during head-up tilt testing even in the absence of hypotension or tachycardia: a quantitative, controlled study using Doppler echography. Clin Neurophysiol Pract 2020; 5: 50-58. 